\title{
CARACTÉRISATION DE LA CERCAIRE DE BUCEPHALUS POLYMORPHUS BAER, 1827 (TREMATODA, BUCEPHALIDAE) : chétotaxie et système excréteur
}

\author{
M. WALLET et A. LAMBERT*
}

RÉSUMÉ. Nous décrivons le système excréteur et la chétotaxie de la cercaire de Bucephalus polymorphus Baer, 1827 qui se développe chez Dreissena polymorpha (Lamellibranche, Dreissenidae) dans le sud-est de la France. Nous comparons nos observations avec celles réalisées en Pologne et discutons des différences observées.

\section{Characterization of the cercaria of Bucephalus polymorphus Baer, 1827 (Trematoda, bucephalidae) : chetotaxy and excretory system}

SUMMARY. We describe the excretory system and the chetotaxy of the cercaria of Bucephalus polymorphus Baer, 1827 which develops in Dreissena polymorpha (Lamellibranch, Dreissenidae) in South-East of France. We compare our observations with those realised in Poland and we discuss about the differences observed.

On a longtemps confondu sous la dénomination Bucephalus polymorphus Baer, 1827 plusieurs espèces de Bucephalidae d'eau douce. Baturo (1977-1979) après un travail expérimental montre que cette dénomination doit se limiter aux cercaires gastérostomes qui se développent chez Dreissena polymorpha. Quant à celles qui se développent chez Unio pictorum elles donnent des adultes de Rhipidocotyle illense (Ziegler, 1883).

Bucephalus polymorphus ayant été signalé comme exerçant une action pathogène vis-à-vis du Cyprinidae deuxième hôte intermédiaire (De Kinkelin, Besse, Tuffery, 1968) il était nécessaire de caractériser cette cercaire avant d'entamer toute étude épidémiologique sur la parasitose qu'elle peut engendrer.

\footnotetext{
* Laboratoire de Parasitologie comparée, ERA C.N.R.S. 915, Université des Sciences et Techniques du Languedoc, Place E.-Bataillon, F 34060 Montpellier Cedex.

Ce travail a bénéficié de l'aide financière du Conseil Supérieur de la Pêche (convention C.S.P.-U.S.T.L. $\mathrm{n}^{\circ}$ 82.316).

Accepté le 30 janvier r 984 .
} 


\section{Matériel et méthodes}

A partir d'individus de Dreissena polymorpha qui émettent des cercaires gastérostomes, nous avons vérifié expérimentalement, après infestation de Cyprinidae (Phoxinus phoxinus) que les métacercaires intramusculaires qui se sont formées, donnent chez de jeunes Sandres (Lucioperca lucioperca) des adultes de Bucephalus polymorphus.

Les cercaires ont été étudiées in vivo, entre lame et lamelle pour décrire l'appareil excréteur. Les imprégnations argentiques ont été réalisées directement sans fixation préalable, selon la technique de Combes, Bayssade-Dufour et Cassone (1976).

La nomenclature adoptée est celle de Richard (1971) et Bayssade-Dufour (1979).

Si la morphologie générale de la cercaire correspond à la description donnée par Baturo (1977), il n'en est pas de même pour le système excréteur et la chétotaxie.

\section{Le système excréteur}

La formule excrétrice générale est : $2[(3+3+3)+(3+3+3)]=36$, mais des variations de détail peuvent exister : ainsi il est possible d'observer une quatrième cellule flamme dans certains groupes.

De chaque côté du corps, les canaux collecteurs antérieur et postérieur forment un tronc collecteur commun débouchant dans la région latéro-postérieure d'une vessie tubulaire, sinueuse, qui s'étend du pharynx à l'extrémité postérieure du corps.

\section{La chétotaxie (fig. 1 à 6 )}

Bayssade-Dufour (1979) a discuté les problèmes que posait la nomenclature des papilles chez les cercaires gastérostomes où la bouche ne s'ouvre pas dans la région céphalique mais au niveau de l'acétabulum ventral. Nous décrivons ici la chétotaxie selon cette interprétation :

1 - Papilles buccales (fig. 2 et 5 )

$\mathrm{C}_{\mathrm{I}}=6 \mathrm{C}_{\mathrm{I}} \mathrm{V}+2 \mathrm{C}_{\mathrm{I}} \mathrm{L}+3 \mathrm{C}_{\mathrm{I}} \mathrm{D}$

$\mathrm{C}_{\mathrm{II}}=2 \mathrm{C}_{\mathrm{II}} \mathrm{V}+1 \mathrm{C}_{\mathrm{II}} \mathrm{L}+1 \mathrm{C}_{\mathrm{II}} \mathrm{D}$

$\mathrm{C}_{\mathrm{III}}=4 \mathrm{C}_{\mathrm{III}} \mathrm{V}+6 \mathrm{C}_{\mathrm{III}} \mathrm{L}+5 \mathrm{C}_{\mathrm{III}} \mathrm{D}$

Il existe 2 papilles internes dans le rhynchus.

2 - Papilles corporelles (fig. 1 et 4)

$\mathrm{A}_{\mathrm{I}}=2 \mathrm{~A}_{\mathrm{I}} \mathrm{V}+3$ à $4 \mathrm{~A}_{\mathrm{I}} \mathrm{L}+1 \mathrm{~A}_{\mathrm{I}} \mathrm{D}$

$\mathrm{A}_{\text {II }}=2 \mathrm{~A}_{\mathrm{II}} \mathrm{V}+0 \mathrm{~A}_{\mathrm{II}} \mathrm{L}+1 \mathrm{~A}_{\mathrm{II}} \mathrm{D}$

$\mathrm{A}_{\text {III }}=0$ à $1 \mathrm{~A}_{\text {III }} \mathrm{V}+3$ à $4 \mathrm{~A}_{\text {III }} \mathrm{L}+1 \mathrm{~A}_{\mathrm{III}} \mathrm{D}$

$\mathrm{M}=2 \mathrm{MV}+0 \mathrm{ML}+1 \mathrm{MD}$

$\mathrm{P}_{\mathrm{I}}=0$ à $1 \mathrm{P}_{\mathrm{I}} \mathrm{V}+0 \mathrm{P}_{\mathrm{I}} \mathrm{L}+0 \mathrm{P}_{\mathrm{I}} \mathrm{D}$

$\mathrm{P}_{I I}=0 \mathrm{P}_{I I} \mathrm{~V}+3 \mathrm{P}_{I I} \mathrm{~L}+0 \mathrm{P}_{I I} \mathrm{D}$

$\mathrm{P}_{I I I}=0 \mathrm{P}_{I I I} \mathrm{~V}+3 \mathrm{P}_{I I I} \mathrm{~L}+0 \mathrm{P}_{I I I} \mathrm{D}$ 


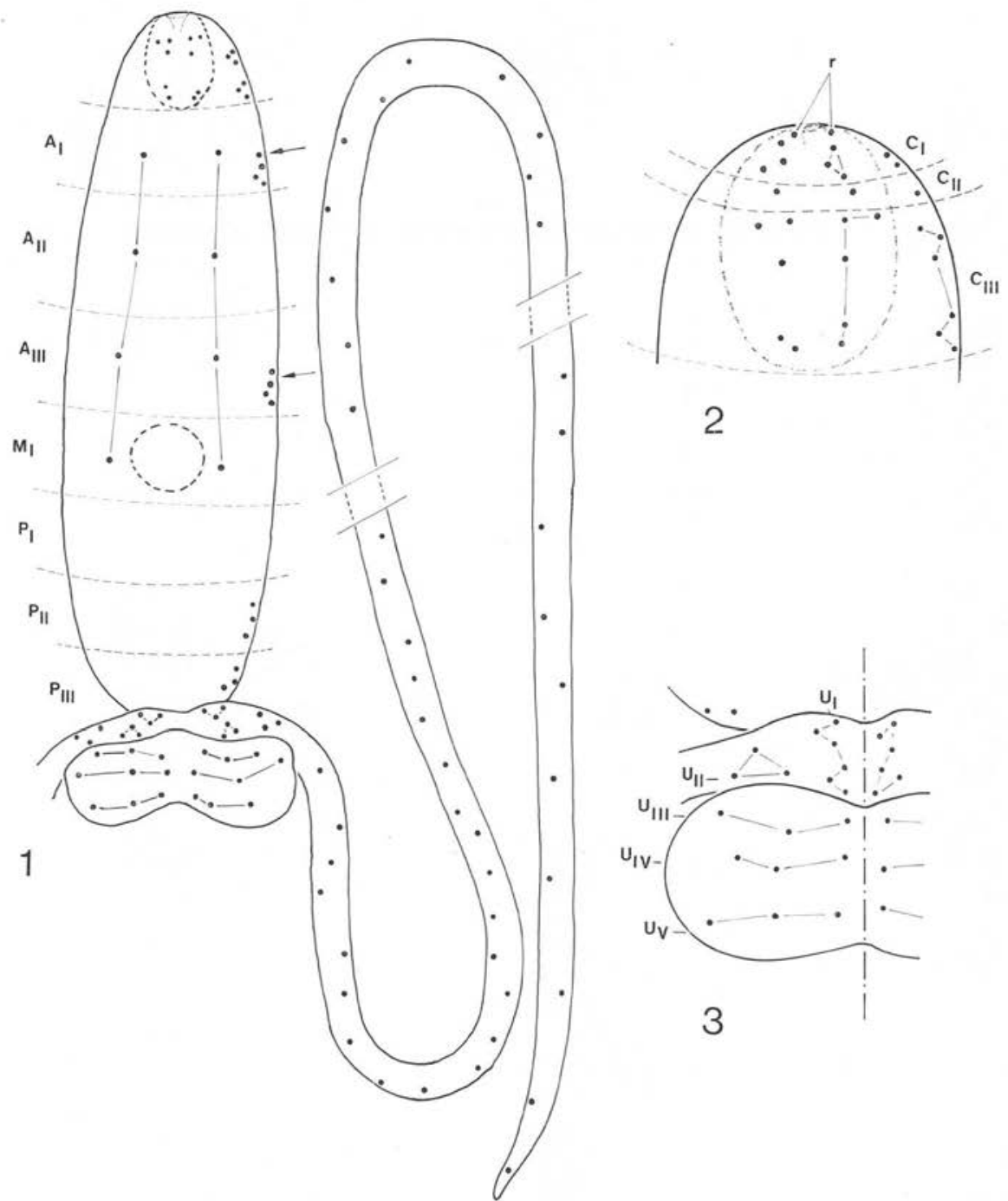

FIG. I, 2 et 3. - Chétotaxie de la cercaire de Bucephalus polymorphus Baer, I827: face dorsale.

Fig. $I$ : papilles corporelles; fig. 2 : papilles céphaliques. $r$ : papilles internes du rhynchus; fig. $3:$ papilles caudales.

(Les papillles latérales sout représentées sur le côté droit du corps. Les flèches indiquent d'éventuelles papilles supplémentaires,) 


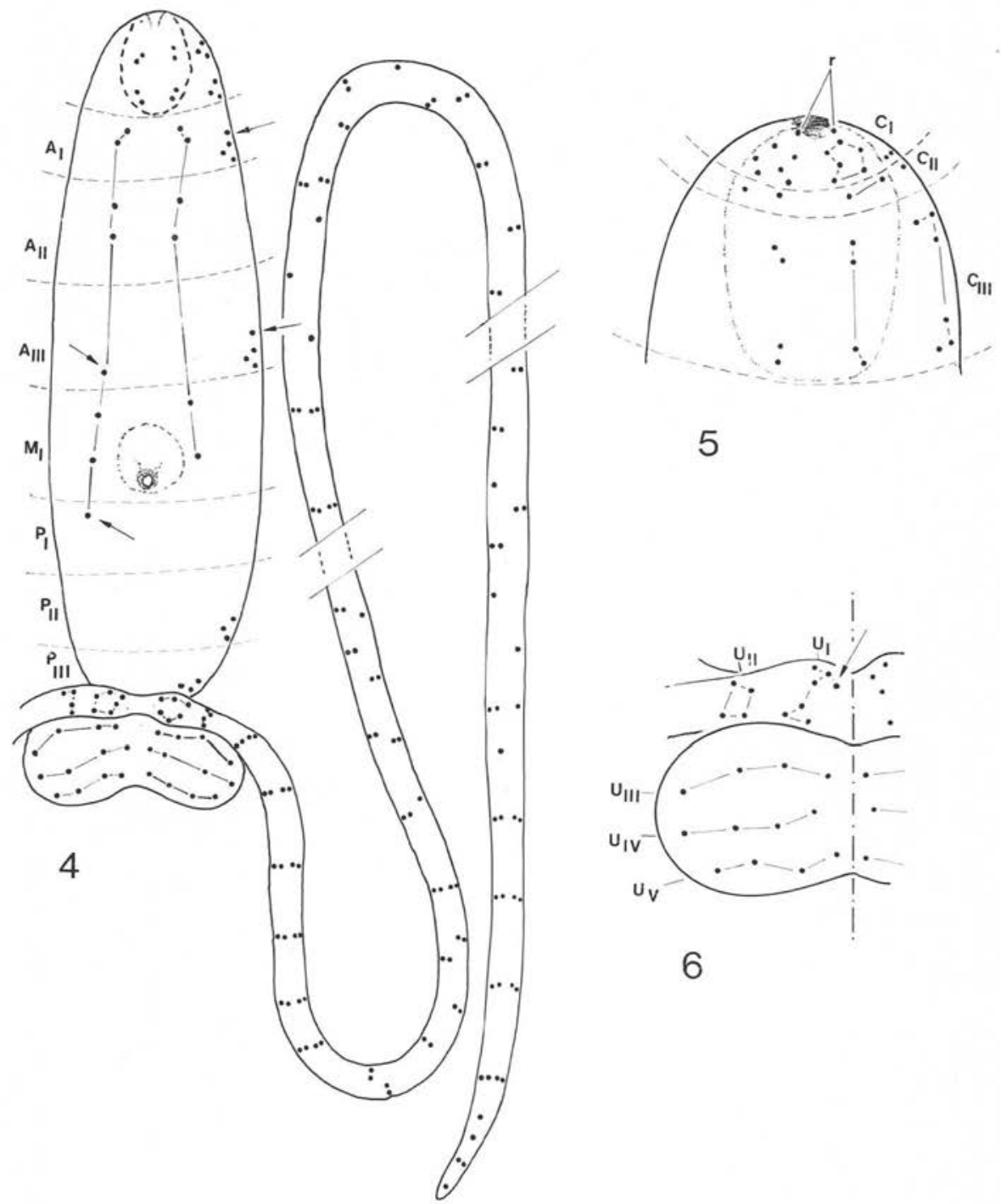

Fig. 4, 5 et 6. - Chétotaxie de la cercaire de Bucephalus polymorphus Baer, 1827 : face ventrale.

Fig. 4: papilles corporelles; fig. 5 : papilles céphaliques. $\mathrm{r}$ : papilles internes du rhynchus ; fig. 6: papilles caudales.

(Les papilles latérales sont représentées sur le côté gauche du corps. Les flèches indiquent d'éventuelles papilles supplémentaires.) 
3 - Papilles caudales ( fig. 3 et 6 )

$\begin{array}{lll}\mathrm{U}_{\mathrm{IV}}: 6 \text { à } 7 & \mathrm{U}_{\mathrm{I}} \mathrm{D}: 6 \\ \mathrm{U}_{\mathrm{II}} \mathrm{V}: 4 & \mathrm{U}_{\mathrm{II}} \mathrm{D}: 3 \\ \mathrm{U}_{\mathrm{III}} \mathrm{V}: 4 & \mathrm{U}_{\mathrm{III}} \mathrm{D}: 3 \\ \mathrm{U}_{\mathrm{IV}} \mathrm{V}: 4 & \mathrm{U}_{\mathrm{IV}} \mathrm{D}: 3 \\ \mathrm{U}_{\mathrm{V} V}: 4 & \mathrm{U}_{\mathrm{V} D}: 3\end{array}$

4 - Papilles des fourchons caudaux ( fig. 1 et 6 )

Ventralement, il y a généralement 2 rangs de papilles doubles faisant suite aux cycles $\mathrm{U}_{\mathrm{I}} \mathrm{V}$ et $\mathrm{U}_{\mathrm{II}} \mathrm{V}$, où peuvent s'intercaler des papilles impaires. On compte en tout 70 à 80 doublets de sensilles.

Dorsalement, faisant suite aux cycles $\mathrm{U}_{\mathrm{I}} \mathrm{D}$ et $\mathrm{U}_{\mathrm{II}} \mathrm{D}$, il y a un rang de 80 à 90 papilles impaires.

\section{Conclusion}

Nos observations sur la morphologie et la biologie de la cercaire de Bucephalus polymorphus confirment en général celles de Baturo (1977). Nous avons noté des différences quant à l'organisation du système excréteur : Baturo donne : $2[(4+4+4+4)+(4+4+4)]=56$ protonéphridies alors que nous avons établi la formule : $2[(3+3+3)+(3+3+3)]=36$ (il est parfois possible d'observer pour chacun de ces groupes une protonéphridie supplémentaire). L'absence d'un groupe de cellules flammes nous paraît être un argument suffisant pour ne pas rejeter l'hypothèse de races géographiques, hypothèse d'autant plus intéressante à vérifier que Bucephalus polymorphus peut engendrer ou non des phénomènes pathogènes au niveau des Cyprinidae deuxième hôte intermédiaire. Notons que cette deuxième formule se rapproche de celles observées chez la plupart des Bucephalidae décrits (Combes et coll., 1980).

La description trop sommaire de la chétotaxie donné par Baturo ne permet malheureusement pas une comparaison précise avec nos observations. La seule cercaire de Bucephalidae connue par sa chétotaxie est celle de Labratrema lamirandi, Bucephalidae marin (Bayssade-Dufour et Maillard, 1975) qui se développe chez Cardium glaucum, mais il est prématuré sur la base de ces seuls exemples de détailler une comparaison qui ferait la part des affinités attestant l'appartenance à une famille et la part des différences dont il restera à préciser les origines : de ce point de vue l'étude de la chétotaxie des Bucephalidae d'eau douce apporterait beaucoup car, si les cercaires de Bucephalus polymorphus et de Rhipidocotyle illense sont toutes deux de type gastérostome, elles correspondent à deux modes très différents de transmission : infestation active pour la première, infestation passive pour la seconde (Baturo, 1977). Il serait alors intéressant de comparer la chétotaxie, d'une part chez Labratrema lamirandi et Bucephalus polymorphus où les cercaires s'enkystent après 
pénétration active au travers de la peau et de la musculature, d'autre part chez Rhipidocotyle illense où la cercaire se comporte comme un élément planctonique qui est entraîné dans le courant d'eau respiratoire. Il y a peut-être là un modèle qui permettrait de mettre en évidence d'éventuelles variations adaptatives de l'équipement sensoriel des cercaires, liées à l'écologie de la transmission.

\section{BIBLIOGRAPHIE}

BAturo B. : Bucephalus polymorphus Baer, 1827 and Rhipidocotyle illense (Ziegler, I883) (Trematoda Bucephalidae) : morphology and biology of developmental stages. Acta Parasit. Polonica, $1977,24,203-220$.

BAturo B. : Bucephalus Baer, 1827 and B. polymorphus Baer, 1827 (Trematoda) proposed use of the plenary powers to conserve these name in accordance with general use. Bull. Zool. Nomenclature, $1979,36,30-36$.

Bayssade-Dufour C., MAILlard C. : Chétotaxie de Labratrema lamirandi (Carrere, 1937) Maillard 1975, Bucephaloidea. Observations sur Cercaria plumosa Sinitzin ror I, Fellodistomatoidea. Comparaison avec Cercaria sp. Bayssade-Dufour 1974, Brachylaimoidea. Discussions sur le sous-ordre des Brachylaimata La Rue, 1957 (Trematoda). Bull. Mus. Nat. Hist. Nat., Paris, $3^{\mathrm{e}}$ série, $\mathrm{n}^{\circ} 283$, 1975, Zool. 193, 39-68.

BAYSSADE-DUFOUR C. : L'appareil sensoriel des cercaires et la systématique des Trématodes digénétiques. Mem. Mus. Nat. Hist. Nat., 1979, série A, Zool. II3, I-81.

Combes C., Albaret J.-L., Arvy L., Bartoli P., Bayssade-Dufour C., Deblock S., DuretteDesset M.-C., Gabrion C., Jourdane J., Lambert A., Leger N., Maillard C., Matricon M., Nassi H., Prevot G., Richard J., Theron A. : Atlas Mondial des Cercaires, Mem. Mus. Nat. Hist. Nat., I980, série A, Zool., IIS, 235 p.

Combes C., BAyssade-Dufour C., CASSOne J. : Sur l'imprégnation et le montage des cercaires pour l'étude chétotaxique. Ann. Parasit. Hum. Comp., I976, 51, 399-400.

DE Kinkelin P., Besse P., Tuffery G. : Une nouvelle affection nécrosante des téguments et des nageoires : la Bucéphalose larvaire à Bucephalus polymorphus Baer, 1827. Bull. Off. Int. Epiz., 1968, 69, 1207-1230.

RichaRD J. : La chétotaxie des cercaires. Valeur systématique et phylétique. Mem. Mus. Nat. Hist. Nat., I971, Sér. A, Zool., 67, I-179. 